\title{
Half of Canadians can expect cancer diagnosis during lifetime
}

\author{
Cite as: CMAJ 2017 July 10;189:E920. doi: 10.1503/cmaj.1095447
}

$\mathbf{N}$ early half of Canadians will get cancer during their lifetime, according to Canadian Cancer Statistics 2017 . The risk is $49 \%$ for men and $45 \%$ for women, states the report by the Canadian Cancer Society. Cancer is the leading cause of death in Canada, responsible for one in four deaths.

"These numbers are an important reminder of the challenge we continue to face with cancer, despite the progress we've made," said Leah Smith, an epidemiologist with the Canadian Cancer Society. "Cancer takes a huge toll, not only on health care resources, but on Canadians and their loved ones. We have to take action to reduce this risk. Prevention, support and research are key."

These figures are relatively the same as last year, when the society reported that "almost half of all Canadians will develop cancer in their lifetime, and one quarter of Canadians are expected to die of the disease."

According to the new report, about 206200 people are expected to be diagnosed with cancer in 2017, up from the 202400 diagnoses expected last year. Nearly $90 \%$ of cases will be among people age 50 and older (similar to last year's figure of $89 \%$ ). About 80800 Canadians will die from the disease, compared to 78800 in 2016. The number of cases is expected to rise as Canada's population continues to age.

The two most common types of cancer diagnosed overall are colorectal (13\%) and lung cancer (14\%). Breast cancer is the most common among women, affecting one in eight. Prostate cancer is the most common among men, affecting one

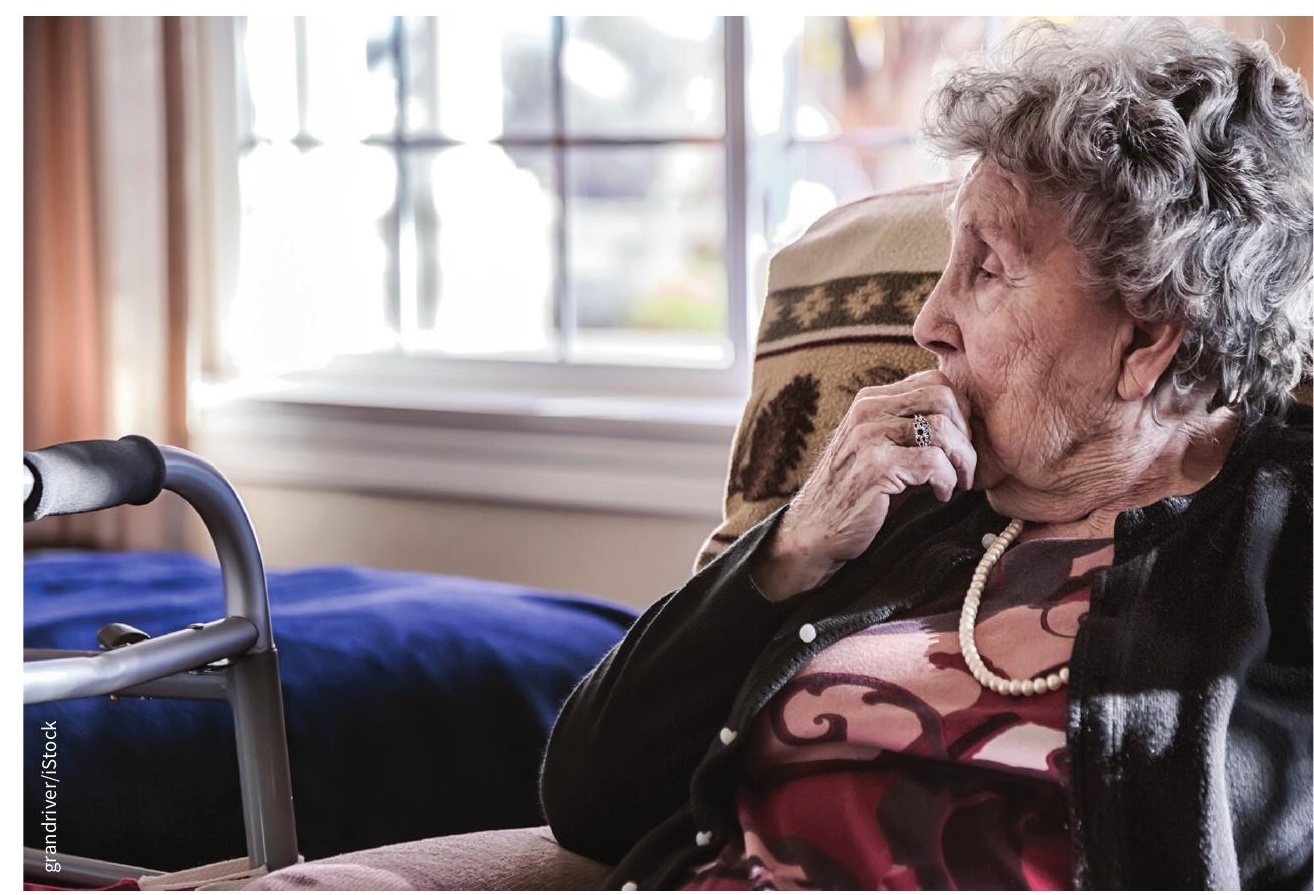

Cancer rates expected to continue rising as population ages.

in seven. Lung, breast, colorectal and prostate cancers accounted for half of all cancer cases in 2016.

One worrying finding, according to Smith, is that pancreatic cancer may soon become the third-leading cause of cancer death in the country. There will be an estimated 5500 diagnoses of this cancer in 2017 and 4800 deaths.

"Many of us are surprised to learn that because pancreatic cancer isn't a cancer we hear about a lot," said Smith. "The reality is pancreatic cancer has the lowest survival rate of the 23 cancers we reported on, and we've made very little progress with this disease, especially relative to the other major cancers. Since little is known about preventing pancreatic cancer and detecting it early, research is key to making a difference with this disease."

Overall, however, advances in cancer research have improved survival rates considerably, according to the report. In the 1940s, the five-year survival rate was only $25 \%$. It has increased to $60 \%$. An estimated 179000 cancer deaths have been avoided since 1988 thanks to improvements in cancer prevention and control.

Canadian Cancer Statistics 2017 was produced by the Canadian Cancer Society, the Public Health Agency of Canada and Statistics Canada.

\section{Roger Collier, CMAJ}

